\title{
Birth weight alters the response to postnatal high-fat diet-induced changes in meat quality traits and skeletal muscle proteome of pigs
}

\author{
Jingbo Liu, Jun He, Jie Yu, Xiangbing Mao, Ping Zheng, Zhiqing Huang, Bing Yu and Daiwen Chen* \\ Institute of Animal Nutrition, Sichuan Agricultural University, No. 46, Xinkang Road, Ya'an, Sichuan 625014, \\ People's Republic of China
}

(Submitted 22 August 2013 - Final revision received 22 November 2013 - Accepted 16 December 2013 - First published online 30 January 2014)

\begin{abstract}
Low birth weight (LBW) exerts persistent effects on the growth and development of offspring. The present study was conducted to test the hypothesis that LBW alters the response of pigs to high-fat (HF) diet-induced changes in meat quality and skeletal muscle proteome. Normalbirth weight (NBW) and LBW piglets were fed a control diet or a HF diet from weaning to slaughter at 110 kg body weight. Most of the meat quality traits were influenced by LBW. Meat quality analysis revealed that LBW piglets had a greater ability to deposit intramuscular lipids than their heavier littermates when fed a HF diet. Increased shear force, lower $\mathrm{pH}_{45}$ min and drip loss were observed in the skeletal muscle of LBW piglets compared with NBW piglets. Proteomic analysis revealed forty-six differentially expressed proteins in the skeletal muscle of LBW and NBW piglets fed the control diet or HF diet. These proteins play a central role in cell structure and motility, glucose and energy metabolism, lipid metabolism, and cellular apoptosis, as well as stress response. Of particular interest is the finding that LBW altered the response to HF diet-induced changes in the expression of proteins related to stress response (heat shock protein) and glucose and energy metabolism (pyruvate kinase, phosphoglycerate mutase, enolase and triosephosphate isomerase). Taken together, our findings revealed that the HF diet-induced changes in the expression of glucose and energy metabolism-related proteins varied between NBW and LBW piglets, which provides a possible mechanism to explain higher intramuscular fat store in LBW pigs when fed a HF diet.
\end{abstract}

Key words: Birth weight: High-fat diet: Pigs: Proteome: Skeletal muscle

Epidemiological studies have demonstrated an association between slow growth of fetus in utero and a greater risk of CHD, type 2 diabetes, the metabolic syndrome and osteoporosis in later life ${ }^{(1,2)}$. Previous studies on fetal programming have suggested that the pattern of mammalian embryo or fetus development is programmed by the in utero experience to match the anticipated postnatal life, thus increasing the fitness of the offspring ${ }^{(3)}$. However, the programmed phenotype may induce harmful effects on the adaptability of the offspring when the practically experienced environment of the offspring is not consistent with the predicted postnatal environment ${ }^{(4)}$.

Intrauterine growth retardation is a condition where the fetus does not reach its growth potential during pregnancy, thus leading to the low birth weight (LBW) of offspring ${ }^{(5)}$. Skeletal muscle plays an important role in the process of metabolic diseases ${ }^{(6)}$. The results of a previous study have revealed that LBW impairs the expression of proteins involved in nutrient metabolism, immune response, cell structure and antioxidant function in the skeletal muscle of newborn piglets ${ }^{(7)}$. There is extensive evidence that LBW changes the response of the offspring to postnatal environment, nutrition and stress reflected by the mRNA expression levels of key transcriptional factors, hormone secretion, nutrient metabolism and lipid deposition ${ }^{(8-11)}$. High-fat (HF) diet feeding is a wellcharacterised experimental model that is used for medical and nutritional studies ${ }^{(12)}$. Nevertheless, whether the postnatal HF diet-induced changes in the expression of proteins in the skeletal muscle are dependent on the development of the offspring programmed by the in utero environment is scarcely understood. Furthermore, available data on fetal programming have been obtained mainly in rodents and not in pigs. Therefore, the present study was conducted to test whether the effect of a postnatal HF diet on meat quality traits and skeletal muscle proteome may differ between LBW and normal-birth weight (NBW) pigs. The results of the present study may provide a feeding strategy to reveal the detrimental effects of LBW on growth performance and meat quality.

\section{Materials and methods}

\section{Animals and diets}

The animal protocol of the present study was approved by the Animal Care and Use Committee of Sichuan Agricultural

Abbreviations: C, control diet; CHAPS, 3-[(3-cholamidopropyl)dimethylammonio]propanesulfonic acid; HF, high fat; HSP, heat shock protein; IMF, intramuscular fat; LBW, low birth weight; NBW, normal birth weight; SM, semitendinosus muscle. 
University. A total of forty cross-bred male piglets (Duroc (Landrace $\times$ Yorkshire)) from twenty litters were used in the present study (one NBW piglet and one LBW piglet were selected per litter). The body weight of each piglet was recorded at birth. Piglets with a birth weight above the average litter birth weight were defined as NBW piglets and those with a birth weight 2 standard deviations lower than the average litter birth weight were identified as LBW piglets. From weaning (day 28) to slaughter at approximately $110 \mathrm{~kg}$ body weight (Table 1), twenty NBW and twenty LBW male piglets were fed either a control diet (C, without lard supplementation) or a HF diet (supplemented with $10 \%$ lard), thus forming four experimental groups (birth weight/ diet): NBW/C; NBW/HF; LBW/C; LBW/HF ( $n$ 10). Dietary protein levels were decreased over the growth period to meet the optimal growth requirements of pigs. Piglets were penned individually in metabolic cages with woven wire flooring and were given ad libitum access to feed (at 08.00, 14.00 and 20.00 hours) and water. The feed intake of piglets was calculated weekly, and the body weight of piglets was recorded monthly.

\section{Sample collection and meat quality analysis}

All the piglets were slaughtered when their body weight was approximately $110 \mathrm{~kg}$ (NBW piglets on day 154 and LBW piglets on day 178). Piglets were killed by electrical stunning and exsanguination after an overnight fast. Immediately after slaughter, the whole semitendinosus muscle (SM) was collected from the right side of the carcass. SM samples collected for proteomic analysis were frozen in liquid $\mathrm{N}_{2}$ and stored at $-80^{\circ} \mathrm{C}$. The $L^{*}$ (lightness), $a^{*}$ (redness) and $b^{*}$ (yellowness) values of the SM were determined using Minolta Chromameter CR-300 (Minolta, Inc.) according to pork colour standards (Japanese Color Standards). Drip loss in the SM was determined according to the difference in sample weight after suspension at $4^{\circ} \mathrm{C}$ for $24 \mathrm{~h}$. The Warner-Bratzler shear force was used to assess the tenderness of the skeletal muscle as described previously ${ }^{(13)}$. The cooking loss of SM was determined using a water-bath at $70^{\circ} \mathrm{C}$ after vacuum packaging. Furthermore, $3 \mathrm{~g} \mathrm{SM}$ sample was homogenised in $5 \mathrm{ml}$ of pure water, and the $\mathrm{pH}$ values were measured using a pH metre (PHS-3D; Shanghai REX Instrument Factory) at $45 \mathrm{~min}$ and $24 \mathrm{~h}$ post-mortem. Intramuscular fat (IMF) content in the SM was determined using the chloroform-methanol extraction method ${ }^{(14)}$.

\section{Two-dimensional difference gel electrophoresis}

Skeletal muscle samples (approximately $0.2 \mathrm{~g}$ ) were ground into powder using a precooled mortar and pestle in liquid $\mathrm{N}_{2}$. The samples were then homogenised in a lysis buffer (7 M-urea, $2 \mathrm{M}$-thiourea and 4\% 3-((3-cholamidopropyl) dimethylammonio)propanesulphonic acid (CHAPS) and $50 \mathrm{~mm}$-dithiothreitol containing $1 \%$ protease inhibitors) using a glass homogeniser. The fractions were fully suspended,

Table 1. Composition of the experimental diets

\begin{tabular}{|c|c|c|c|c|c|c|c|c|}
\hline \multirow[b]{3}{*}{ Dietary levels of fat... } & \multicolumn{8}{|c|}{ Diet feeding period } \\
\hline & \multicolumn{2}{|c|}{$5-8$ weeks } & \multicolumn{2}{|c|}{$9-12$ weeks } & \multicolumn{2}{|c|}{$13-20$ weeks } & \multicolumn{2}{|c|}{21 weeks-110 kg } \\
\hline & $\mathrm{C}$ & $\mathrm{HF}$ & $\mathrm{C}$ & $\mathrm{HF}$ & $\mathrm{C}$ & $\mathrm{HF}$ & $\mathrm{C}$ & $\mathrm{HF}$ \\
\hline \multicolumn{9}{|l|}{ Ingredients (\%) } \\
\hline Maize & $49 \cdot 50$ & $49 \cdot 50$ & $56 \cdot 27$ & $56 \cdot 27$ & 61.65 & 61.65 & $64 \cdot 38$ & $64 \cdot 38$ \\
\hline Decupled soyabean meal & $13 \cdot 50$ & $13 \cdot 50$ & & & & & & \\
\hline Soyabean meal & & & $23 \cdot 55$ & 23.55 & $18 \cdot 50$ & $18 \cdot 50$ & $16 \cdot 60$ & $16 \cdot 60$ \\
\hline Maize starch & $10 \cdot 00$ & & $10 \cdot 00$ & & $10 \cdot 00$ & & $10 \cdot 00$ & \\
\hline Lard & & $10 \cdot 00$ & & $10 \cdot 00$ & & $10 \cdot 00$ & & $10 \cdot 00$ \\
\hline Fishmeal & $2 \cdot 00$ & $2 \cdot 00$ & 2.50 & 2.50 & $2 \cdot 50$ & 2.50 & $2 \cdot 00$ & $2 \cdot 00$ \\
\hline Whey powder & $10 \cdot 00$ & $10 \cdot 00$ & & & & & & \\
\hline Bran & & & $5 \cdot 00$ & $5 \cdot 00$ & $5 \cdot 00$ & $5 \cdot 00$ & $5 \cdot 00$ & $5 \cdot 00$ \\
\hline Plasma protein & $2 \cdot 00$ & $2 \cdot 00$ & & & & & & \\
\hline Soyabean protein concentrate & $10 \cdot 00$ & $10 \cdot 00$ & & & & & & \\
\hline $\mathrm{NaCl}$ & 0.25 & 0.25 & 0.25 & 0.25 & 0.25 & 0.25 & 0.25 & 0.25 \\
\hline Choline chloride & $0 \cdot 10$ & $0 \cdot 10$ & $0 \cdot 10$ & $0 \cdot 10$ & 0.10 & $0 \cdot 10$ & 0.10 & 0.10 \\
\hline Limestone & 0.80 & 0.80 & 0.60 & 0.60 & 0.60 & 0.60 & 0.60 & 0.60 \\
\hline Dicalcium phosphate & $1 \cdot 20$ & $1 \cdot 20$ & $1 \cdot 10$ & $1 \cdot 10$ & 0.80 & 0.80 & 0.50 & 0.50 \\
\hline L-Lys & 0.15 & 0.15 & 0.13 & 0.13 & 0.10 & 0.10 & 0.07 & 0.07 \\
\hline Vitamin and mineral premix ${ }^{*}$ & 0.50 & 0.50 & 0.50 & 0.50 & 0.50 & 0.50 & 0.50 & 0.50 \\
\hline Total & $100 \cdot 00$ & $100 \cdot 00$ & $100 \cdot 00$ & $100 \cdot 00$ & $100 \cdot 00$ & $100 \cdot 00$ & $100 \cdot 00$ & $100 \cdot 00$ \\
\hline \multicolumn{9}{|l|}{ Calculated nutrient and energy levels } \\
\hline Metabolisable energy (MJ/kg) & $14 \cdot 22$ & $15 \cdot 96$ & $13 \cdot 77$ & $15 \cdot 50$ & $13 \cdot 83$ & $15 \cdot 57$ & $13 \cdot 89$ & $15 \cdot 62$ \\
\hline Crude protein (\%) & $20 \cdot 05$ & $20 \cdot 02$ & 18.05 & $18 \cdot 02$ & $16 \cdot 05$ & $16 \cdot 02$ & $15 \cdot 04$ & $15 \cdot 01$ \\
\hline $\mathrm{Ca}(\%)$ & 0.90 & 0.90 & 0.74 & 0.74 & 0.65 & 0.65 & 0.56 & 0.56 \\
\hline Total P (\%) & 0.67 & 0.67 & 0.63 & 0.63 & 0.55 & 0.55 & 0.48 & 0.48 \\
\hline Available P (\%) & 0.48 & 0.48 & 0.39 & 0.39 & 0.34 & 0.34 & 0.27 & 0.27 \\
\hline Digestible Lys (\%) & $1 \cdot 10$ & $1 \cdot 10$ & 1.00 & 1.00 & 0.84 & 0.84 & 0.75 & 0.75 \\
\hline
\end{tabular}

C, control diet (without lard supplementation); HF, high-fat diet (supplemented with $10 \%$ lard).

*Provided per kg of diet: vitamin $A, 1.90 \mathrm{mg}$; vitamin $D_{3}, 0.06 \mathrm{mg}$; vitamin $E, 24 \mathrm{mg}$; vitamin $\mathrm{K}_{3}, 3 \mathrm{mg}$; vitamin $\mathrm{B}_{1}, 1.5 \mathrm{mg}$; vitamin $\mathrm{B}_{2}, 6 \mathrm{mg}$; vitamin $\mathrm{B}_{6}, 3 \mathrm{mg}$; vitamin $\mathrm{B}_{12}, 0.024 \mathrm{mg}$; nicotinic acid, $20 \mathrm{mg}$; pantothenic acid, $15 \mathrm{mg}$; biotin, $0.15 \mathrm{mg}$; folic acid, $1.2 \mathrm{mg} ; \mathrm{Fe}\left(\mathrm{FeSO}_{4} .7 \mathrm{H}_{2} \mathrm{O}\right), 120 \mathrm{mg} ; \mathrm{Cu}\left(\mathrm{CuSO}_{4} .5 \mathrm{H}_{2} \mathrm{O}\right)$,

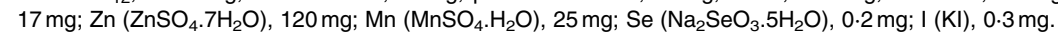


sonicated on ice and centrifuged at $40000 \boldsymbol{g}$ for $15 \mathrm{~min}$. The supernatants were collected, and protein concentrations were measured using the Bradford method (Bio-Rad Laboratories, Inc. $)^{(15)}$. Protein extracts were stored at $-80^{\circ} \mathrm{C}$ until use.

The $\mathrm{pH}$ of the protein extracts was adjusted to 8.5 by addition of $50 \mathrm{~mm}-\mathrm{NaOH}$. Pooled samples were used to reduce the costs of the experiment. For pooling, equal amounts of total protein from three different piglets were combined randomly within the groups. Because nine piglets were selected from each treatment group, three biological replicates per treatment group were used in the proteomic analysis. Equal amounts of proteins from the twelve samples ( $n 3$ ) were pooled together to produce the internal standards. The skeletal muscle samples were labelled with Cy3 or Cy5, whereas the internal standards were labelled with Cy2 using $400 \mathrm{pmol}$ of fluorochrome $/ 50 \mu \mathrm{g}$ of protein. Cy2, Cy3 and Cy5 were purchased from GE Healthcare. Labelling was carried out for $30 \mathrm{~min}$ on ice in the dark. From each treatment, $50 \mathrm{mg}$ Cy3- and Cy5-labelled samples were combined before mixing with a Cy2-labelled internal standard, and an equal volume of $2 \times$ sample buffer ( $7 \mathrm{M}$-urea, $2 \mathrm{M}$-thiourea, $4 \%$ CHAPS, $1 \%$ Pharmalyte (pH 3-10) and $20 \mathrm{mg} / \mathrm{ml}$ dithiothreitol) was added. The rehydration buffer ( $7 \mathrm{M}$-urea, $2 \mathrm{M}$-thiourea, $4 \%$ CHAPS, $0.5 \%$ Pharmalyte and $10 \mathrm{mg} / \mathrm{ml}$ dithiothreitol) was added to the samples, and the total volume was made up to $410 \mu \mathrm{l}$.

The samples were actively rehydrated into $24 \mathrm{~cm} \mathrm{3-10} \mathrm{NL}$ immobilised $\mathrm{pH}$ gradient strips for $12 \mathrm{~h}$ at $17^{\circ} \mathrm{C}$ using an IPGphor (GE Healthcare). Isoelectric focusing was conducted for a total of $80000 \mathrm{Vh}$ (ramp to $250 \mathrm{~V}$ in $30 \mathrm{~min}$, hold at $1000 \mathrm{~V}$ for $1 \mathrm{~h}$, ramp to $10000 \mathrm{~V}$ in $5 \mathrm{~h}$, hold at 10000 for $60000 \mathrm{Vh}$ ). The immobilised $\mathrm{pH}$ gradient strips were equilibrated in an equilibration buffer (6 m-urea, 2\% SDS, 50 mm-Tris ( $\mathrm{pH} \mathrm{8.8)}$ and $30 \%$ glycerol) containing $0.5 \%$ dithiothreitol for $15 \mathrm{~min}$ followed by equilibration in $4.5 \%$ iodoacetamide in the equilibration buffer for $15 \mathrm{~min}$ incubation at room temperature. The immobilised $\mathrm{pH}$ gradient strips were placed on the top of $12 \%$ homogeneous polyacrylamide gels. The seconddimension SDS-PAGE was carried out using Ettan DALT Six electrophoresis units (GE Healthcare).

\section{Image analysis}

After SDS-PAGE, the gels were scanned using Typhoon 9410 scanner (GE Healthcare) with Ettan DALT Gel Alignment Guides at excitation/emission wavelengths specific for Cy2 $(488 \mathrm{~nm} / 520 \mathrm{~nm})$, Cy3 $(532 \mathrm{~nm} / 580 \mathrm{~nm})$ and Cy5 (633 nm/ $670 \mathrm{~nm})$. The range of the intensity was adjusted within 60000-90000 pixel value to ensure a maximum volume for each image. The differential in-gel electrophoresis images were analysed using the DeCyder version 6.5 software (GE Healthcare) according to the manufacturer's protocol. All thirty-six protein spot maps from the twelve gels were simultaneously matched and confirmed manually. The expression of proteins was analysed using two-way ANOVA. Protein spots that were differentially expressed between the groups ( $\mid$ ratiol $\geq 1.2$ and $P \leq 0.05$ ) were marked and selected for identification.

\section{Protein identification and database search}

Protein spots of interest were obtained with preparative gels. Electrophoresis was carried out as described above, except that $500-1000 \mu \mathrm{g}$ of protein were loaded in the immobilised $\mathrm{pH}$ gradient strips and the gels were stained with Coomassie Brilliant Blue. The selected protein spots were manually obtained and destained with $25 \mathrm{~mm}$-ammonium bicarbonate and $50 \%$ acetonitrile for $1 \mathrm{~h}$. After drying the gels by centrifugal lyophilisation, the protein samples were digested with $0.01 \mu \mathrm{g} / \mu \mathrm{l}$ trypsin (Promega) in $25 \mathrm{~mm}$-ammonium bicarbonate at $37^{\circ} \mathrm{C}$ for $15 \mathrm{~h}$. The resulting peptides were subjected to sequential extraction with $5 \%$ trifluoroacetic acid at $40^{\circ} \mathrm{C}$ for $1 \mathrm{~h}$ and with $2.5 \%$ trifluoroacetic acid and $50 \%$ acetonitrile at $30^{\circ} \mathrm{C}$ for $1 \mathrm{~h}$. The extract samples were dried by centrifugal lyophilisation.

Peptide mixtures were mixed with a matrix solution (4-hydroxy- $\alpha$-cyanocinnamic acid in 30\% acetonitrile and $0 \cdot 1 \%$ trifluoroacetic acid). Matrix-assisted laser desorption ionisation-time-of-flight/time-of-flight MS (MALDI-TOF/TOF) analysis was carried out on the 4800 Proteomics Analyzer (Applied Biosystems). After MS, parent mass peaks with a mass range of $600-4000 \mathrm{Da}$ and a minimum signal:noise ratio of 15 were selected for MS/MS analysis. Peptide mass fingerprint and MS/MS data searches were carried out for protein identification using the GPS Explorer ${ }^{\mathrm{TM}}$ software (Applied Biosystems) with MASCOT search program (Matrix Science) and the search taxonomy of Mammalia against the NCBI database. The following parameters were included for the database search: trypsin as the cleaving enzyme; a maximum of one missed trypsin cleavage; both peptide and MS/MS tolerance of $0.2 \mathrm{Da}$; monoisotopic as mass value; oxidation and carbamidomethyl as variable modifications. Confident identification was based on the protein score. A protein score $>67$ was considered to be statistically significant $(P<0.05)$.

\section{Statistical analysis}

Statistical analysis was carried out using the general linear model procedure of SAS (SAS Institute). The model included the effects of birth weight (NBW or LBW), postnatal diet ( $\mathrm{C}$ or $\mathrm{HF}$ ) and the interactions between them. The effect of litter was included as a random factor in this model. Growth performance and meat quality trait data are presented as means with their standard errors. Differences were considered significant at $P<0.05$.

\section{Results}

\section{Growth performance}

The birth weight and weaning weight of LBW piglets were lower than those of their NBW littermates $(P<0 \cdot 01$; Table 2$)$. From weaning to slaughter at $110 \mathrm{~kg}$ body weight, the average daily feed intake and average daily gain were lower in LBW piglets than in NBW piglets $(P<0 \cdot 01)$, while the feed:gain ratio was higher in LBW piglets than in NBW piglets $(P<0.05$; Table 2$)$. HF diet feeding increased the average 
Table 2. Effects of birth weight (BW) and postnatal high-fat (HF) diet (supplemented with $10 \%$ lard) on the growth performance of piglets from weaning to slaughter at $110 \mathrm{~kg}$ body weight

(Mean values with their standard errors; $n$ 10)

\begin{tabular}{|c|c|c|c|c|c|c|c|c|}
\hline \multirow[b]{2}{*}{ Items } & \multicolumn{2}{|c|}{ NBW } & \multicolumn{2}{|c|}{ LBW } & \multirow[b]{2}{*}{ SEM } & \multicolumn{3}{|c|}{$P$} \\
\hline & C & $\mathrm{HF}$ & C & $\mathrm{HF}$ & & BW & Diet & I \\
\hline BW (kg) & 1.78 & 1.72 & 0.95 & 0.95 & 0.03 & $<0.01$ & NS & NS \\
\hline Weaning weight $(\mathrm{kg})$ & 8.86 & 8.89 & $5 \cdot 68$ & 5.40 & 0.41 & $<0.01$ & NS & NS \\
\hline Average daily gain $(\mathrm{g} / \mathrm{d})$ & 801 & 892 & 671 & 725 & $10 \cdot 7$ & $<0.01$ & $<0.01$ & NS \\
\hline Average daily feed intake $(\mathrm{g} / \mathrm{d})$ & 2113 & 1878 & 1806 & 1595 & 35.4 & $<0.01$ & $<0.01$ & NS \\
\hline Feed:gain ratio & 2.64 & $2 \cdot 11$ & 2.69 & $2 \cdot 20$ & 0.03 & $<0.05$ & $<0.01$ & NS \\
\hline
\end{tabular}

C, control diet (without lard supplementation); NBW, normal birth weight; LBW, low birth weight; I, interaction.

daily gain and reduced the average daily feed intake and the feed:gain ratio $(P<0 \cdot 01$; Table 2$)$.

\section{Meat quality traits}

The effects of birth weight and HF diet on meat quality traits are summarised in Table 3. The $\mathrm{pH}_{45}$ min, drip loss and lightness of SM were lower in LBW piglets than in NBW piglets $(P<0 \cdot 01)$. The IMF content, redness, yellowness and Warner-Bratzler shear force of SM were higher in LBW piglets than in NBW piglets $(P<0 \cdot 01)$. The IMF content, lightness and yellowness of SM were higher in piglets fed the HF diet than in those fed the $\mathrm{C}$ diet $(P<0 \cdot 01)$. The IMF and cooking loss were affected by the interaction of birth weight and postnatal diet $(P<0.05)$.

\section{Skeletal muscle proteome}

In the present study, about 2000 spots were automatically detected on the gels. A total of forty-six proteins were differentially expressed in the skeletal muscle of LBW and NBW piglets fed the $\mathrm{C}$ diet or the HF diet postnatally (Table 4). The positions of these protein spots in the gel image are shown in Fig. 1. According to their biological function, these differentially expressed proteins were classified into seven groups: (1) cell structure and motility; (2) glucose and energy metabolism; (3) lipid metabolism; (4) stress response;
(5) protein and amino acid metabolism; (6) cell redox homeostasis; (7) cellular apoptosis.

\section{Cell structure and motility}

A total of twenty-six protein spots were related to cell structure and motility. These were myosin heavy chain (spots 58 , 122, 320, 415, 609, 614, 799, 813, 841, 858 and 1278), myosin-binding protein C (spot 69), intermediate filament desmin (spot 672), $\alpha$-actin (spot 745), troponin T fast skeletal muscle type (spots 817 and 849), troponin T1 slow skeletal muscle type (spots 1027 and 1030), troponin I (spot 1378), capping protein (spot 832), tropomyosin (spots 932, 1024 and 1148) and fast skeletal myosin alkali light chain (spots 1314, 1661 and 1680). The abundance of myosin heavy chain $(P<0.05)$, myosin-binding protein $\mathrm{C}(P<0.05)$, intermediate filament desmin $(P<0.01), \alpha$-actin $(P<0.05)$ and troponin T1 slow skeletal muscle type $(P<0.05)$ was lower in the skeletal muscle of LBW piglets in that of NBW piglets. By contrast, the expression of troponin $\mathrm{T}$ fast skeletal muscle type $(P<0.05)$, troponin I $(P<0.05)$ and capping protein $(P<0.05)$ was up-regulated in LBW piglets. HF diet feeding resulted in a lower expression of myosin heavy chain $(P<0.05)$, troponin $\mathrm{T}$ fast skeletal muscle type $(P<0.05)$ and tropomyosin $(P<0.05)$ and a higher expression of fast skeletal myosin alkali light chain $1(P<0.05)$ compared with $\mathrm{C}$ diet feeding. Furthermore, the expression of myosin heavy chain, capping protein and fast skeletal myosin alkali light chain 1

Table 3. Effects of birth weight (BW) and postnatal high-fat (HF) diet (supplemented with $10 \%$ lard) on meat quality traits of piglets

(Mean values with their standard errors; $n$ 10)

\begin{tabular}{|c|c|c|c|c|c|c|c|c|}
\hline \multirow[b]{2}{*}{ Items } & \multicolumn{2}{|c|}{ NBW } & \multicolumn{2}{|c|}{ LBW } & \multirow[b]{2}{*}{ SEM } & \multicolumn{3}{|c|}{$P$} \\
\hline & C & $\mathrm{HF}$ & C & $\mathrm{HF}$ & & BW & Diet & I \\
\hline $\mathrm{pH}_{45 \text { min }}$ & $6 \cdot 73$ & $6 \cdot 73$ & 6.38 & $6 \cdot 51$ & 0.04 & $<0.01$ & NS & NS \\
\hline $\mathrm{pH}_{24 \mathrm{~h}}$ & $5 \cdot 62$ & $5 \cdot 63$ & $5 \cdot 58$ & $5 \cdot 64$ & 0.04 & NS & NS & NS \\
\hline IMF (\%) & 4.58 & 4.37 & 5.36 & 6.04 & 0.25 & $<0.01$ & $<0.01$ & $<0.05$ \\
\hline Lightness $\left(L^{*}\right)$ & 45.00 & $46 \cdot 33$ & 38.76 & 41.03 & 0.48 & $<0.01$ & $<0.01$ & NS \\
\hline Redness $\left(a^{*}\right)$ & $7 \cdot 68$ & $10 \cdot 11$ & 14.94 & 15.79 & 0.85 & $<0.01$ & NS & NS \\
\hline Yellowness $\left(b^{\star}\right)$ & 2.86 & 3.47 & 4.34 & 4.80 & 0.19 & $<0.01$ & $<0.01$ & NS \\
\hline Cooking loss (\%) & $35 \cdot 16$ & 37.51 & 37.71 & 33.88 & 1.01 & NS & NS & $<0.05$ \\
\hline Drip loss (\%) & 3.87 & 4.27 & 1.86 & 1.52 & 0.24 & $<0.01$ & NS & NS \\
\hline WBSF $(\mathrm{kg})$ & 5.69 & 5.81 & 6.36 & 6.41 & $0 \cdot 19$ & $<0.01$ & NS & NS \\
\hline
\end{tabular}

NBW, normal birth weight; LBW, low birth weight; C, control diet (without lard supplementation); l, interaction; IMF, intramuscular fat; WBSF, Warner-Bratzler shear force. 
Table 4. Effects of birth weight (BW) and postnatal high-fat (HF) diet (supplemented with $10 \%$ lard) on the skeletal muscle proteome of piglets*

\begin{tabular}{|c|c|c|c|c|c|c|c|c|c|c|}
\hline \multirow[b]{2}{*}{ Spot number† } & \multirow[b]{2}{*}{ Protein name } & \multirow[b]{2}{*}{ Accession number } & \multirow[b]{2}{*}{ Protein score $\ddagger$} & \multicolumn{2}{|c|}{ NBW } & \multicolumn{2}{|c|}{ LBW } & \multicolumn{3}{|c|}{$P$} \\
\hline & & & & C & $\mathrm{HF}$ & C & $\mathrm{HF}$ & BW & Diet & l \\
\hline \multicolumn{11}{|c|}{ Cell structure and motility } \\
\hline 58 & Myosin, heavy chain 4 , skeletal muscle (Sus scrofa) & gi|178056718 & 319 & 1.00 & $-1 \cdot 18$ & -1.44 & -1.78 & $<0.05$ & NS & NS \\
\hline 69 & Myosin-binding protein C, fast-type isoform 2 (Mus musculus) & gi|22122731 & 115 & 1.00 & 1.06 & -1.81 & $-2 \cdot 41$ & $<0.05$ & NS & NS \\
\hline 122 & Myosin, heavy chain 4 , skeletal muscle (S. scrofa) & gi|178056718 & 99 & 1.00 & $-1 \cdot 21$ & -1.25 & -1.56 & $<0.01$ & $<0.05$ & NS \\
\hline 320 & Myosin, heavy chain 1 , skeletal muscle, adult (S. scrofa) & gi|157279731 & 409 & 1.00 & $-1 \cdot 30$ & -1.91 & -1.85 & $<0.05$ & NS & NS \\
\hline 415 & Myosin, heavy chain 1 , skeletal muscle, adult ( $S$. scrofa) & gi|157279731 & 307 & 1.00 & $-1 \cdot 10$ & -1.44 & $-1 \cdot 19$ & $<0.05$ & NS & NS \\
\hline 609 & Myosin, heavy chain 1 , skeletal muscle, adult (S. scrofa) & gi|157279731 & 113 & 1.00 & $-1 \cdot 27$ & -1.52 & $-2 \cdot 11$ & $<0.05$ & NS & NS \\
\hline 614 & Myosin, heavy chain 4 , skeletal muscle (S. scrofa) & gi|178056718 & 112 & 1.00 & $1 \cdot 16$ & -1.60 & -1.52 & $<0.05$ & NS & NS \\
\hline 799 & Myosin, heavy chain 1 , skeletal muscle, adult (S. scrofa) & gi|157279731 & 135 & 1.00 & 1.24 & 1.35 & -1.51 & NS & $<0.05$ & $<0.05$ \\
\hline 813 & Myosin, heavy polypeptide 2 , skeletal muscle, adult (S. scrofa) & gi|55741490 & 93 & 1.00 & $-1 \cdot 14$ & -1.95 & -1.60 & $<0.05$ & NS & NS \\
\hline 841 & Myosin, heavy chain 1 , skeletal muscle, adult (S. scrofa) & gi|157279731 & 93 & 1.00 & $-1 \cdot 23$ & -1.22 & 1.02 & NS & NS & $<0.01$ \\
\hline 858 & Myosin, heavy chain 1 , skeletal muscle, adult ( $S$. scrofa) & gi|157279731 & 155 & 1.00 & 1.42 & $1 \cdot 19$ & 1.01 & NS & NS & $<0.05$ \\
\hline 1278 & Myosin heavy chain (S. scrofa) & gi|1431613 & 131 & 1.00 & $-1 \cdot 24$ & -1.88 & -1.76 & $<0.05$ & NS & NS \\
\hline 672 & Muscle-specific intermediate filament desmin (S. scrofa) & gi|48374063 & 136 & 1.00 & -1.03 & -1.44 & $-1 \cdot 21$ & $<0.01$ & NS & NS \\
\hline 745 & $\alpha$-Actin (Homo sapiens) & gi|178027 & 254 & 1.00 & -1.09 & $-2 \cdot 27$ & -1.36 & $<0.05$ & NS & NS \\
\hline 817 & Troponin T fast skeletal muscle type (S. scrofa) & gi|46389787 & 97 & 1.00 & $-1 \cdot 10$ & 1.91 & $1 \cdot 17$ & $<0.05$ & NS & NS \\
\hline 849 & Troponin $T$ fast skeletal muscle type (S. scrofa) & gi|46389785 & 112 & 1.00 & -1.32 & 1.41 & 1.04 & $<0.05$ & $<0.05$ & NS \\
\hline 1027 & Troponin T1, skeletal, slow (S. scrofa) & gi|55741809 & 144 & 1.00 & $-1 \cdot 10$ & -1.54 & -1.29 & $<0.05$ & NS & NS \\
\hline 1030 & Troponin T1, skeletal, slow (S. scrofa) & gi|55741809 & 75 & 1.00 & -1.02 & -1.73 & -1.75 & $<0.05$ & NS & NS \\
\hline 1378 & Troponin I (Oryctolagus cuniculus) & gi|130493079 & 79 & 1.00 & 1.00 & 1.88 & 1.43 & $<0.05$ & NS & NS \\
\hline 832 & Capping protein $(H$. sapiens $)$ & gi|433308 & 116 & 1.00 & $1 \cdot 25$ & 1.20 & 1.05 & NS & NS & $<0.05$ \\
\hline 932 & Tropomyosin $2(\beta)$ isoform 1 (H. sapiens) & gi|42476296 & 219 & 1.00 & -1.07 & 1.44 & 1.51 & $<0.05$ & NS & NS \\
\hline 1024 & Striated-muscle $\alpha$-tropomyosin (Rattus norvegicus) & gi|207349 & 804 & 1.00 & -1.53 & $1 \cdot 11$ & -1.52 & NS & $<0.05$ & NS \\
\hline 1148 & TPMsk3 (H. sapiens) & gi|19072649 & 67 & 1.00 & 1.23 & 1.05 & 1.24 & NS & $<0.05$ & NS \\
\hline 1314 & Fast skeletal myosin alkali light chain 1 (S. scrofa) & gi|157427687 & 117 & 1.00 & -1.36 & -1.54 & 1.08 & NS & NS & $<0.05$ \\
\hline 1661 & Fast skeletal myosin alkali light chain 1 (S. scrofa) & gi|157427687 & 413 & 1.00 & 1.34 & 1.26 & $1 \cdot 74$ & NS & $<0.05$ & NS \\
\hline 1680 & Fast skeletal myosin alkali light chain 1 (S. scrofa) & gi|157427687 & 113 & 1.00 & 1.01 & $-1 \cdot 14$ & 1.39 & NS & $<0.05$ & $<0.05$ \\
\hline \multicolumn{11}{|c|}{ Glucose and energy metabolism } \\
\hline 400 & Pyruvate kinase, muscle isoform $\mathrm{M} 1$ ( $H$. sapiens) & gi|33286420 & 580 & 1.00 & $-1 \cdot 13$ & -1.46 & -1.49 & $<0.05$ & NS & NS \\
\hline 436 & Pyruvate kinase, muscle isoform $\mathrm{M} 1$ (H. sapiens) & gi|33286420 & 598 & 1.00 & 1.54 & $1 \cdot 14$ & $-1 \cdot 17$ & NS & NS & $<0.05$ \\
\hline 405 & Phosphoglucomutase 1 (S. scrofa) & gi|3746944 & 105 & 1.00 & -1.56 & -1.40 & -1.98 & $<0.05$ & $<0.05$ & -- \\
\hline 617 & Muscle-specific enolase $\beta$ subunit (M. musculus) & gi|387144 & 68 & 1.00 & 1.02 & -1.29 & 1.13 & NS & $<0.05$ & $<0.05$ \\
\hline 1285 & Phosphoglycerate mutase (H. sapiens) & gi|387016 & 203 & 1.00 & $-1 \cdot 16$ & -1.57 & $-1 \cdot 14$ & $<0.01$ & NS & $<0.01$ \\
\hline 1297 & Triosephosphate isomerase ( $R$. norvegicus) & gi|538426 & 194 & 1.00 & 1.32 & 1.38 & -1.26 & NS & NS & $<0.05$ \\
\hline 1470 & Adenylate kinase 1 ( $H$. sapiens) & gil55958256 & 209 & 1.00 & -1.64 & 1.06 & $-1 \cdot 13$ & NS & $<0.05$ & NS \\
\hline \multicolumn{11}{|l|}{ Lipid metabolism } \\
\hline 1142 & apo B mRNA editing enzyme (Pongo abelii) & gi|197098854 & 105 & 1.00 & $-1 \cdot 20$ & 1.54 & 1.45 & $<0.05$ & - & - \\
\hline 1390 & apo A-I (S. scrofa) & gi|164359 & 115 & 1.00 & $1 \cdot 16$ & 1.21 & 1.76 & $<0.05$ & - & - \\
\hline 1708 & Fatty acid-binding protein 3 (S. scrofa) & gi|50262041 & 110 & 1.00 & $1 \cdot 16$ & 1.31 & 3.05 & $<0.01$ & - & - \\
\hline \multicolumn{11}{|c|}{ Stress response } \\
\hline 278 & Heat shock $70 \mathrm{kDa}$ protein 5 (H. sapiens) & gi|16507237 & 227 & 1.00 & -1.14 & -1.61 & 1.08 & NS & NS & $<0.05$ \\
\hline 1523 & Heat shock protein, $\alpha$-crystallin-related, B6 (H. sapiens) & gi|21389433 & 103 & 1.00 & 2.85 & $2 \cdot 04$ & $2 \cdot 69$ & NS & $<0.05$ & NS \\
\hline 1292 & Heat shock protein $27 \mathrm{kDa}$ (S. scrofa) & gi|50916342 & 117 & 1.00 & -1.07 & $-1 \cdot 11$ & $1 \cdot 23$ & NS & NS & $<0.05$ \\
\hline \multicolumn{11}{|c|}{ Protein and amino acid metabolism } \\
\hline 487 & $26 S$ proteasome non-ATPase regulatory subunit 8 ( $M$. musculus) & gi|20978554 & 67 & 1.00 & $1 \cdot 17$ & 1.07 & $-1 \cdot 30$ & $<0.05$ & NS & $<0.01$ \\
\hline 652 & Leucine aminopeptidase 3 (H. sapiens) & gi|37588925 & 74 & 1.00 & $1 \cdot 30$ & 1.58 & $-1 \cdot 14$ & NS & NS & $<0.05$ \\
\hline
\end{tabular}


was affected by the interaction of birth weight and HF diet $(P<0 \cdot 05)$.

\section{Glucose and energy metabolism}

Differentially expressed proteins involved in glucose and energy metabolism were pyruvate kinase (spots 400 and 436), phosphoglucomutase 1 (spot 405), enolase (spot 617), phosphoglycerate mutase (spot 1285), triosephosphate isomerase (spot 1297) and adenylate kinase 1 (spot 1470). The expression of pyruvate kinase $(P<0.05)$, phosphoglucomutase $1 \quad(P<0.05)$ and phosphoglycerate mutase $(P<0.01)$ was lower in LBW piglets than in NBW piglets. The abundance of enolase was lower and those of phosphoglucomutase 1 and adenylate kinase 1 were higher in piglets fed the HF diet than in those fed the $\mathrm{C}$ diet $(P<0.05)$. In addition, the expression of pyruvate kinase $(P<0.05)$, enolase $(P<0.05)$, phosphoglycerate mutase $(P<0.01)$ and triosephosphate isomerase $(P<0.05)$ was affected by the interaction of birth weight and postnatal HF diet.

\section{Lipid metabolism}

LBW increased the abundance of proteins involved in lipid metabolism $(P<0 \cdot 05)$. These proteins were apo B mRNA editing enzyme (spot 1142), apo A-I (spot 1390) and fatty acid-binding protein 3 (spot 1708).

\section{Stress response}

The expression of heat shock protein (HSP) with different molecular weights related to stress response was affected by birth weight, postnatal diet and the interaction between them. The levels of HSP B6 (spot 1523) tended $(P<0 \cdot 1)$ to be higher in LBW piglets than in NBW piglets, and HF diet feeding resulted in a higher expression of HSP B6 compared with $\mathrm{C}$ diet feeding $(P<0 \cdot 05)$. However, the levels of HSP $70 \mathrm{kDa}$ (spot 278) and HSP $27 \mathrm{kDa}$ (spot 1292) were influenced by the interaction of birth weight and HF diet $(P<0.05)$.

\section{Protein and amino acid metabolism}

A central role is played by $26 \mathrm{~S}$ proteasome non-ATPase regulatory subunit 8 (spot 487 ) in protein degradation. The expression of this protein was influenced by birth weight $(P<0.05)$ and the interaction of birth weight and diet $(P<0.01)$. The abundance of amino acid metabolism-related leucine aminopeptidase 3 (spot 652) was affected by the interaction of birth weight and postnatal diet $(P<0 \cdot 05)$.

\section{Cell redox homeostasis}

Differentially expressed proteins related to cell redox homeostasis were L-lactate dehydrogenase A (spot 1091) and carbonic anhydrase III (spot 1267). The abundance of L-lactate dehydrogenase A was increased, while that of carbonic anhydrase III in LBW piglets was decreased compared with that in NBW piglets $(P<0 \cdot 05)$. 


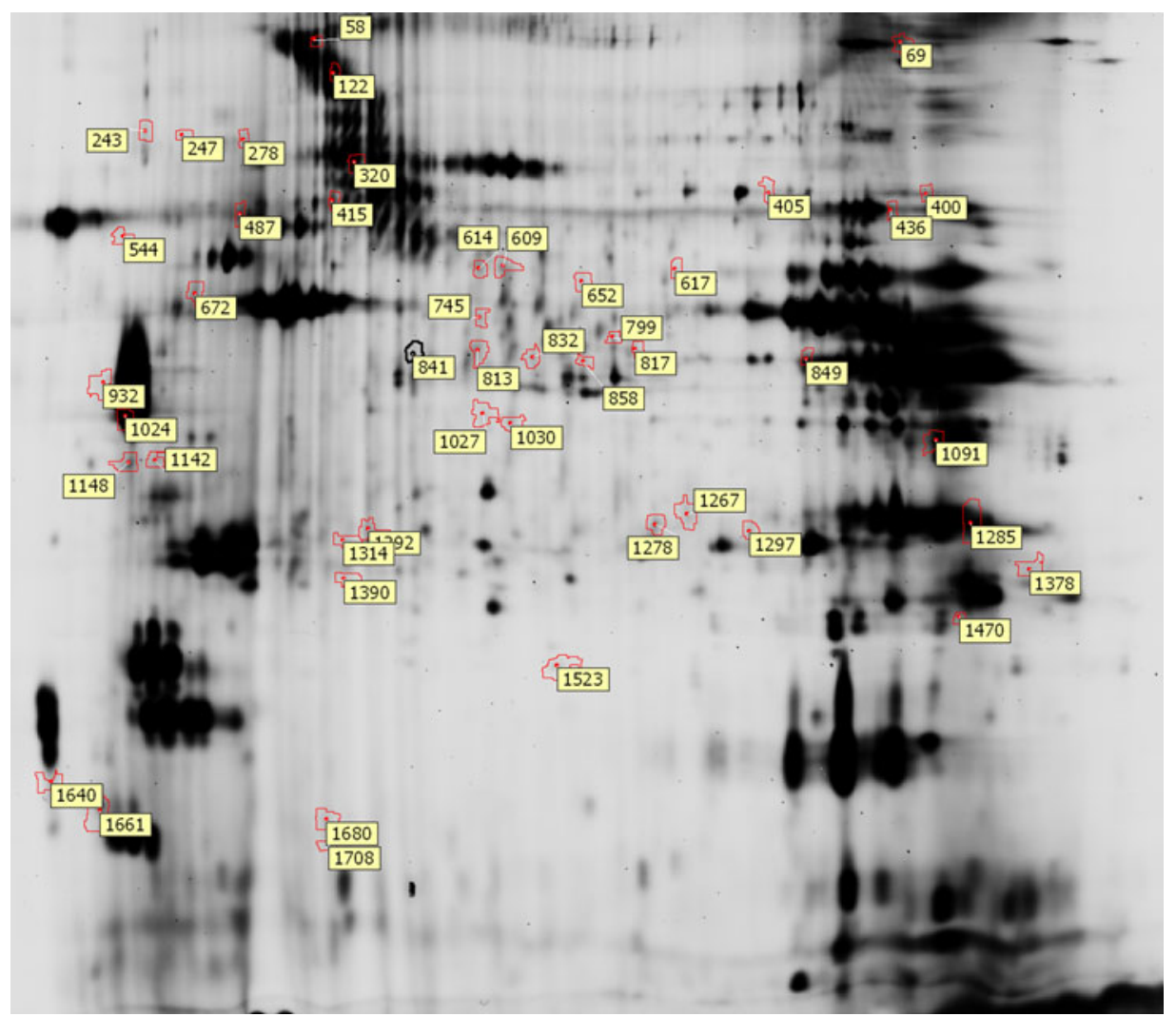

Fig. 1. Two-dimensional differential in-gel electrophoresis image of the of skeletal muscle proteome map of normal-birth weight and low-birth weight piglets ( $n 3$ replicates). The number of identified protein spots was assigned by the analysis software. (A colour version of this figure can be found online at http://www.journals.cambridge.org/bjn)

\section{Cellular apoptosis}

There were three protein spots related to cellular apoptosis. The expression of calreticulin (spot 243), excision repair protein (spot 544) and calmodulin (spot 1640) in LBW piglets was increased compared with that in NBW piglets and was also increased by HF diet feeding $(P<0.05)$. Furthermore, the abundance of excision repair protein was affected by the interaction of birth weight and postnatal diet $(P<0.05)$.

\section{Discussion}

The results of the present study demonstrate that LBW impairs postnatal growth rates and affects meat quality traits ${ }^{(16-18)}$. To test the hypothesis that birth weight alters the response of pigs to postnatal nutrition, we fed LBW and NBW piglets either a $\mathrm{C}$ diet or a HF diet from weaning to slaughter at $110 \mathrm{~kg}$ body weight. The data revealed that LBW piglets had a greater ability to deposit intramuscular lipids than NBW piglets when fed a HF diet. Compared with a previous study that reported that birth weight induced marked changes in skeletal muscle proteome in the newborn piglets ${ }^{(7)}$, novel findings of the present study suggest that these effects are extended to the period when pigs weigh $110 \mathrm{~kg}$. However, the effects of LBW on skeletal muscle proteome in newborn piglets were significantly different from those in piglets with a body weight of $110 \mathrm{~kg}$.

Insufficient intake of nutrients in the fetus is considered to be the main reason for LBW and is commonly associated with low growth rates during postnatal period because of reduced feed intake ${ }^{(5)}$. In the present study, LBW piglets exhibited marked decreases in feed intake and daily gain, suggesting that their appetite was impaired by fetal undernutrition experience, which is consistent with the findings of previous studies $^{(16-18)}$. Increased lipid contents and greater adipocyte diameters in adipose tissues and skeletal muscle were observed in LBW pigs compared with NBW pigs ${ }^{(19,20)}$. A higher proportion of small adipocytes in LBW pigs is suggestive of prolonged adipocyte hyperplasia ${ }^{(20)}$. Consistent with the results of previous studies ${ }^{(10,18)}$, IMF contents were increased in LBW piglets, especially when fed the HF diet. This is in agreement with fetal programming research suggesting that fetal undernutrition is related to increased fat deposition ${ }^{(3)}$. In the present study, we found the shear force of SM to be greater in LBW piglets than in NBW piglets. Indeed, the diameter of myofibres plays a central role in meat tenderness ${ }^{(21)}$. Thus, differences in muscle tenderness between LBW and NBW piglets were expected because myofibres with an enlarged cross-sectional area were observed in LBW piglets compared with their heavier littermates ${ }^{(21)}$. The $\mathrm{pH}_{45}$ min and drip loss in the SM were lower in LBW piglets than in NBW piglets, which is consistent with the findings of a previous study ${ }^{(18)}$.

Proteomics technologies facilitate the analysis of thousands of proteins, thereby providing powerful tools for nutritional and physiological research in pigs ${ }^{(22-25)}$. In the present study, two-dimensional differential in-gel electrophoresis and 
matrix-assisted laser desorption ionisation-time-of-flight/ time-of-flight MS (MALDI-TOF/TOF) analysis were used to investigate the variability in the response of LBW and NBW pigs to postnatal HF diet-induced alterations in skeletal muscle proteome. Using proteomic technology, we identified forty-six differentially expressed proteins that were affected in the skeletal muscle of piglets with different birth weights fed a $\mathrm{C}$ diet or a HF diet. The proteins that were affected were found to be involved in cell structure and motility, glucose and energy metabolism, lipid metabolism, cellular apoptosis and stress response.

Myosin heavy chains, actin, connectin and nebulin account for $80 \%$ of the total protein in the skeletal muscle ${ }^{(26)}$. Therefore, post-mortem degradation of these proteins is important for the meat tenderisation process. Using proteomic technology, twenty-seven differentially expressed proteins related to meat quality were identified in the skeletal muscle of pigs with different shear force ${ }^{(27)}$. A negative correlation between myosin heavy chain and actin expression and shear force and a positive correlation between myosin light chain expression and shear force were established after the analysis of the relationship between protein abundance and shear force of the SM. In the present study, a higher shear force and a lower expression of myosin heavy chains and actin were observed in the SM of LBW piglets. This is consistent with the results reported by Lametsch et $a l .{ }^{(27)}$, who suggested that decreased degradation rates of myosin heavy chains delay meat tenderisation process and then increase shear force ${ }^{(27)}$. Troponin controls the $\mathrm{Ca}^{2+}$-dependent muscle constriction by binding to tropomyosin. The relationship between postmortem troponin $\mathrm{T}$ degradation and meat tenderisation has been established in previous studies ${ }^{(28,29)}$. The expression of troponin T1 slow skeletal muscle type was negatively correlated with drip $\operatorname{loss}^{(30)}$. In the present study, together with reduced drip loss in the SM, a decreased abundance of troponin T1 slow skeletal muscle type and an increased expression of troponin $\mathrm{T}$ fast skeletal muscle type were detected in LBW piglets. It is worth noting that the effects of birth weight on troponin $\mathrm{T}$ varied in different types of muscle, reflected by the increased abundance of troponin $\mathrm{T}$ fast skeletal muscle type and reduced expression of troponin T1 slow skeletal muscle type in LBW piglets compared with NBW littermates. Our finding that the birth weight of pigs had different effects on the expression of the same protein in different muscle types has not been reported in previous studies. The physiological functions vary between different muscle types and may provide a possible explanation for this observation. However, further studies are needed to test our hypothesised mechanism. Although the shear force of the muscle was affected by capping protein ${ }^{(30)}$, the expression of this protein was not affected by birth weight, suggesting that the abundance of capping protein has negligible influence on meat tenderness.

Fatty acid-binding protein is required for the transportation of fatty acids into membranes or mitochondria and for the oxidation of fatty acids or the formation of TAG and phospholipids ${ }^{(31)}$. Indeed, the positive correlation between the levels of fatty acid-binding protein and the number of adipocytes and fat content in pork has been found in a previous study $^{(32)}$. In general, pigs with a higher IMF content have a greater expression of fatty acid-binding protein ${ }^{(33)}$. In agreement with those of previous studies, the present results demonstrate that LBW increases intramuscular lipid content and fatty acid-binding protein expression. As an important component of HDL, apo is derived primarily from the liver and responsible for the transportation of lipids and stabilisation of lipoprotein structure ${ }^{(34)}$. In the present study, LBW piglets had higher levels of apo A than NBW piglets, which suggests that increased amounts of lipids are transported into the skeletal muscle for IMF deposition. In addition, reduced expression of apo A in the jejunum of LBW piglets on days 1, 7 and 21 of life was observed using proteomic analysis, which may be caused by insufficient milk consumption and contribute to impaired digestion, absorption and transport $^{(8)}$

Epidemiological studies have shown that the abnormal energy metabolism of LBW offspring contributes to the increasing risks of developing the metabolic syndrome ${ }^{(1)}$. There were significant differences in the abundance of proteins related to energy metabolism in the skeletal muscle and intestine between the LBW and NBW pigs ${ }^{(7,8)}$. Pyruvate kinase plays a vital role in the utilisation of glucose for the production of acetyl CoA, which is important for ATP synthesis $^{(35)}$. In the present study, LBW piglets had lower levels of pyruvate kinase in the skeletal muscle than NBW piglets, which suggests that the abnormal growth experience of the fetus in utero induces long-term effects on the glycolytic process. Furthermore, the expression of energy metabolismrelated phosphoglucomutase and phosphoglycerate mutase was also decreased in LBW piglets. Adenylate kinase catalyses ATP and AMP to form ADP ${ }^{(36)}$. Enolase converts 2-phosphorylglyceric acid into phosphoenolpyruvic acid ${ }^{(37)}$ Triosephosphate isomerase is required for the synthesis of 3-phosphoglyceraldehyde ${ }^{(38)}$, which is an intermediate substrate in glycolysis. Moreover, HF diet feeding increased the expression of triosephosphate isomerase in NBW piglets, whereas the levels of this enzyme in LBW piglets fed the HF diet were reduced compared with those in LBW piglets fed the $\mathrm{C}$ diet. The expression responses of energy metabolismrelated enzymes to HF diet feeding varied between LBW and NBW piglets. Our observations revealed that NBW piglets adapted to the HF diet by increasing their energy metabolism, whereas the levels of energy metabolism-related enzymes were reduced in LBW piglets fed the HF diet, thus making LBW piglets more susceptible to the effects of HF diet feeding than NBW piglets. This is consistent with the concepts of fetal programming, which suggest that LBW impairs the metabolism of energy in the offspring, especially when fed a high-energy $\operatorname{diet}^{(12)}$.

The enzymatic activity of L-lactate dehydrogenase affects post-mortem lactic acid production. Increased lactic acid production is often related to a rapid decline in $\mathrm{pH}^{(16)}$. In the present study, LBW piglets had a greater abundance of L-lactate dehydrogenase than NBW piglets, which could contribute to lower $\mathrm{pH}_{45}$ min in the muscle of LBW piglets compared with NBW piglets. Calreticulin is a $\mathrm{Ca}^{2+}$-binding protein that is 
responsible for protein folding and transport ${ }^{(39)}$. Calmodulin is a $\mathrm{Ca}^{2+}$-regulated enzyme that participates in inflammation responses, cell metabolism and muscle constriction ${ }^{(40)}$. Consistent with a previous study showing that LBW increases the expression of calreticulin in the jejunum of pigs ${ }^{(8)}$, greater abundances of calreticulin and calmodulin were observed in the skeletal muscle of LBW piglets than in that of NBW piglets in the present study. Taken together, we suggest that birth weight has persistent effects on cellular concentrations of $\mathrm{Ca}^{2+}$, which is a second messenger that affects the signal transduction process, thereby regulating muscle constriction $^{(39)}$.

The biological functions of HSP involve the maintenance of cell homeostasis, repairing of damage, stabilisation of unfolded proteins, remodelling of denatured proteins and avoidance of protein aggregation ${ }^{(41)}$. The expression levels of HSP are stimulated by oxidative stress. In the present study, NBW piglets fed the HF diet and LBW piglets exhibited a greater expression of HSP B6 than NBW piglets fed the C diet. Our observation may be explained by the fact that $\mathrm{HF}$ diets and LBW induced oxidative stress in piglets, thus increasing the need for more HSP for the maintenance of body homeostasis. Reduced expression of HSP is beneficial for improving meat tenderness and favour ${ }^{(42)}$. There was a positive correlation between post-mortem $\mathrm{pH}$ values and HSP expression $^{(43)}$. The tenderness and post-mortem $\mathrm{pH}$ of the muscle were influenced by birth weight, and the expression of HSP was affected by the HF diet and the interaction of birth weight and HF diet in the present study. Excision repair protein is responsible for DNA repair ${ }^{(44)}$. Carbonic anhydrase plays an important role in acid-base homeostasis ${ }^{(45)}$. Leucine aminopeptidase and 26S proteasome non-ATPase regulatory subunit 8 are related to protein degradation ${ }^{(46,47)}$. The expression responses of these proteins suggest that the processes of damage repair, acid-base homeostasis and protein metabolism were affected by birth weight, HF diet and the interaction between them.

In summary, the present study demonstrated that growth performance and meat quality are affected by birth weight. LBW pigs had a greater ability to deposit intramuscular lipids than NBW pigs when fed a HF diet. Proteomic analysis revealed that the birth weight of pigs regulated the expression levels of proteins responsible for meat tenderness, fat deposition and energy metabolism. Birth weight altered the expression responses of proteins involved in energy metabolism and stress to HF diet feeding, which strongly supports the fetal programming idea that fetal development in utero alters the response of offspring to postnatal nutrition-induced changes.

\section{Acknowledgements}

The present study was supported by the national 973 programme of China (2012CB124701) and the earmarked fund for China Agriculture Research System (CARS-36). The national 973 programme of China and the earmarked fund for China Agriculture Research System had no role in the design and analysis of the study or in the writing of this article.
The authors' contributions are as follows: J. L., J. H., J. Y., X. M., P. Z., Z. H., B. Y. and D. C. participated in the experimental design, conducted the research and analysed the data; J. L. and D. C. were responsible for writing the manuscript.

None of the authors has any conflicts of interest to declare.

\section{References}

1. Bruce KD \& Hanson MA (2010) The developmental origins, mechanisms, and implications of metabolic syndrome. J Nutr 140, 648-652.

2. Hales CN \& Barker DJ (2001) The thrifty phenotype hypothesis. Br Med Bull 60, 5-20.

3. Gluckman PD, Hanson MA, Cooper C, et al. (2008) Effect of in utero and early-life conditions on adult health and disease. $N$ Engl J Med 359, 61-73.

4. Godfrey KM, Lillycrop KA, Burdge GC, et al. (2007) Epigenetic mechanisms and the mismatch concept of the developmental origins of health and disease. Pediatr Res 61, R5-R10.

5. Wu G, Bazer FW, Wallace JM, et al. (2006) Board-invited review: intrauterine growth retardation: implications for the animal sciences. J Anim Sci 84, 2316-2337.

6. Kelley DE, Goodpaster B, Wing RR, et al. (1999) Skeletal muscle fatty acid metabolism in association with insulin resistance, obesity, and weight loss. Am J Physiol 277, 1130-1141.

7. Wang J, Chen L, Li D, et al. (2008) Intrauterine growth restriction affects the proteomes of the small intestine, liver, and skeletal muscle in newborn pigs. J Nutr 138, $60-66$

8. Wang X, Wu W, Lin G, et al. (2010) Temporal proteomic analysis reveals continuous impairment of intestinal development in neonatal piglets with intrauterine growth restriction. J Proteome Res 9, 924-935.

9. Vickers MH, Breier BH, Cutfield WS, et al. (2000) Fetal origins of hyperphagia, obesity, and hypertension and postnatal amplification by hypercaloric nutrition. Am J Physiol Endocrinol Metab 279, E83-E87.

10. Liu J, Chen D, Yao Y, et al. (2012) Intrauterine growth retardation increases the susceptibility of pigs to high-fat diet-induced mitochondrial dysfunction in skeletal muscle. PLoS One 7, e34835.

11. Morise A, Sève B, Macé K, et al. (2011) Growth, body composition and hormonal status of growing pigs exhibiting a normal or small weight at birth and exposed to a neonatal diet enriched in proteins. Br J Nutr 105, 1471-1479.

12. Rueda-Clausen CF, Dolinsky VW, Morton JS, et al. (2011) Hypoxia-induced intrauterine growth restriction increases the susceptibility of rats to high-fat diet-induced metabolic syndrome. Diabetes 60, 507-516.

13. Tang RY, Yu B, Zhang KY, et al. (2009) Effects of supplemental magnesium aspartate and short-duration transportation on postmortem meat quality and gene expression of $\mu$-calpain and calpastatin of finishing pigs. Livest Sci 121, 50-55.

14. Folch J, Lees M \& Sloane Stanley GH (1957) A simple method for the isolation and purification of total lipides from animal tissues. J Biol Chem 226, 497-509.

15. Bradford MM (1976) A rapid and sensitive method for the quantitation of microgram quantities of protein utilizing the principle of protein-dye binding. Anal Biochem 72, 248-254.

16. Nissen PM \& Oksbjerg N (2011) Birth weight and postnatal dietary protein level affect performance, muscle metabolism and meat quality in pigs. Animal 5, 1382-1389. 
17. Bee G (2004) Effect of early gestation feeding, birth weight, and gender of progeny on muscle fiber characteristics of pigs at slaughter. J Anim Sci 82, 826-836.

18. Gondret F, Lefaucheur L, Juin H, et al. (2006) Low birth weight is associated with enlarged muscle fiber area and impaired meat tenderness of the longissimus muscle in pigs. J Anim Sci 84, 93-103.

19. Powell SE \& Aberle ED (1981) Skeletal muscle and adipose tissue cellularity in runt and normal birth weight swine. J Anim Sci 52, 748-756.

20. Jones AP \& Friedman MI (1982) Obesity and adipocyte abnormalities in offspring of rats undernourished during pregnancy. Science 19, 1518-1519.

21. Gondret F, Lefaucheur L, Louveau I, et al. (2005) Influence of piglet birth weight on postnatal growth performance, tissue lipogenic capacity and muscle histological traits at market weight. Livest Prod Sci 93, 137-146.

22. Sarr O, Louveau I, Le Huërou-Luron I, et al. (2012) Adipose tissue proteomes of intrauterine growth-restricted piglets artificially reared on a high-protein neonatal formula. J Nutr Biochem 23, 1417-1424.

23. Verma N, Rettenmeier AW \& Schmitz-Spanke S (2011) Recent advances in the use of Sus scrofa (pig) as a model system for proteomic studies. Proteomics 11, 776-793.

24. Sarr O, Louveau I, Kalbe C, et al. (2010) Prenatal exposure to maternal low or high protein diets induces modest changes in the adipose tissue proteome of newborn piglets. J Anim Sci 88, 1626-1641.

25. Liu J, Yao Y, Yu B, et al. (2013) Effect of maternal folic acid supplementation on hepatic proteome in newborn piglets. Nutrition 29, 230-234.

26. Sanger JW, Chowrashi P, Shaner NC, et al. (2002) Myofibrillogenesis in skeletal muscle cells. Clin Orthop Relat Res $\mathbf{4 0 3}$, S153-S162.

27. Lametsch R, Karlsson A, Rosenvold K, et al. (2003) Postmortem proteome changes of porcine muscle related to tenderness. J Agric Food Chem 51, 6992-6997.

28. Ouali A (1992) Proteolytic and physicochemical mechanisms involved in meat texture development. Biochimie $\mathbf{7 4}$, $251-265$.

29. Hopkins DL \& Thompson JM (2002) The degradation of myofibrillar proteins in beef and lamb meat using denaturing electrophoresis - an overview. J Muscle Foods 13, 81-102.

30. Hwang IH, Park BY, Kim JH, et al. (2005) Assessment of postmortem proteolysis by gel-based proteome analysis and its relationship to meat quality traits in pig longissimus. Meat Sci 69, 79-91.

31. Gerbens F, Jansen A \& van Erp AJ (1998) The adipocyte fatty acid-binding protein locus: characterization and association with intramuscular fat content in pigs. Mamm Genome 9, $1022-1026$
32. Damon M, Louveau I, Lefaucheur L, et al. (2006) Number of intramuscular adipocytes and fatty acid binding protein- 4 content are significant indicators of intramuscular fat level in crossbred Large White $\times$ Duroc pigs. J Anim Sci 84, $1083-1092$.

33. Laville E, Sayd T, Terlouw C, et al. (2007) Comparison of sarcoplasmic proteomes between two groups of pig muscles selected for shear force of cooked meat. J Agric Food Chem 55, 5834-5841.

34. Mezdour H, Larigauderie G, Castro G, et al. (2006) Characterization of a new mouse model for human apolipoprotein A-I/C-III/A-IV deficiency. J Lipid Res 47, 912-920.

35. Reynard AM, Hass LF, Jacobsen DD, et al. (1961) The correlation of reaction kinetics and substrate binding with the mechanism of pyruvate kinase. J Biol Chem 236, 2277-2283.

36. Walker JE, Saraste M, Runswick MJ, et al. (1982) Distantly related sequences in the alpha- and beta-subunits of ATP synthase, myosin, kinase and other ATP-requiring enzymes and a common nucleotide binding fold. EMBO J 1, 945-951.

37. Cooper JA, Esch FS, Taylor SS, et al. (1984) Phosphorylation sites in enolase and lactate dehydrogenase utilized by tyrosine protein kinases in vivo and in vitro. J Biol Chem 259, 7835-7841.

38. Rieder SV \& Rose IA (1959) The mechanism of the triosephosphate isomerase reaction. J Biol Chem 234, 1007-1010.

39. Coppolino MG, Woodside MJ, Demaurex N, et al. (1997) Calreticulin is essential for integrin-mediated calcium signalling and cell adhesion. Nature 386, 843-847.

40. Cheung WY (1980) Calmodulin plays a pivotal role in cellular regulation. Science 207, 19-27.

41. Kato K, Ito H \& Inaquma Y (2002) Expression and phosphorylation of mammalian small heat shock proteins. Prog Mol Subcell Biol 28, 129-150.

42. Bernard C, Cassar-Malek I, Le Cunff M, et al. (2007) New indicators of beef sensory quality revealed by expression of specific genes. J Agri Food Chem 55, 5229-5237.

43. Pulford DJ, Fraga Vazquez S, Frost DF, et al. (2008) The intracellular distribution of small heat shock proteins in post-mortem beef is determined by ultimate pH. Meat $S_{c} i$ 79, 623-630.

44. Sekelsky JJ, McKim KS, Chin GM, et al. (1995) The drosophila meiotic recombination gene mei-9 encodes a homologue of the yeast excision repair protein rad1. Genetics 141, 619-627.

45. Lindskog S (1997) Structure and mechanism of carbonic anhydrase. Pharmacol Ther 74, 1-20.

46. Nachlas MM, Crawford DT \& Seligman AM (1957) The histochemical demonstration of leucine aminopeptidase. J Histochem Cytochem 5, 264-278.

47. Ferrell K, Wilkinson CR, Dubiel W, et al. (2000) Regulatory subunit interactions of the $26 \mathrm{~S}$ proteasome, a complex problem. Trends Biochem Sci 25, 83-88. 